\title{
Magnetostrictive Deformation of a Transformer: a Comparison Between Calculation and Measurement
}

\author{
Setareh Gorji Ghalamestani ${ }^{a, 1}$, Lieven Vandevelde ${ }^{a}$, Joris J. J. Dirckx ${ }^{b}$, \\ Patrick Guillaume ${ }^{c}$, and Jan A. A. Melkebeek ${ }^{a}$ \\ ${ }^{a}$ Electrical Energy Laboratory (EELAB), Department of Electrical Energy, \\ Systems and Automation (EESA), \\ Ghent University, Ghent, Belgium \\ ${ }^{b}$ Laboratory of Biomedical Physics, University of Antwerp, Antwerp, Belgium \\ ${ }^{c}$ Acoustics and Vibration Research Group, Department of Mechanical \\ Engineering, Free University of Brussels, Brussels, Belgium
}

\begin{abstract}
Magnetostriction, which refers to the deformation of the core material of transformers and electrical machines, is a main contributor to the total noise. In this work, a technique is proposed to calculate the magnetostrictive deformations of the core of the aforementioned devices. This technique is based on finite element approach with an artificial neural network model of the magnetostrictive behaviour of the core material. The strain measurements are carried out by using a dual heterodyne laser interferometer setup. The proposed technique is then applied to a single-phase test transformer core.

As a validation, the vibration of the magnetised core of the same setup is measured by using a laser scanning vibrometer.
\end{abstract}

Keywords: magnetostriction transformer, finite element, neural network, vibration measurement

\section{Introduction}

Vibrations and noise of electrical machines and especially transformers are becoming increasingly important issue especially when the latter are placed in the residential areas. Transformers are widely used and thus studying their noise contribution is important. The total vibrations and noise are generated in different ways from windings and the core laminations to the tank wall and the fans. Several studies have been reported in the literature on the transformer noise [1], [2], [3].

We focus here only on the core deformation which is produced by magnetostriction. The magnetostriction refers to a three-dimensional deformation of the magnetic material of the core when magnetised. Such deformation produces vibrations and results in an unwanted noise radiation. In general, the magnetostrictive behaviour differs for each material and under every magnetisation. The presence of any external pressure (tensile or compressive

\footnotetext{
${ }^{1}$ Corresponding author. E-mail: Setareh.GorjiGhalamestani@UGent.be
} 
stress) can affect the behaviour. Several magnetostriction strain measurement techniques have been reported in the literature e.g. [4], [5], [6]. For the transformer cores which are mostly built out of electrical steel, the magnetostrictive deformations are in the order of micro meter per meter. As a result, determining the contribution of the magnetostrictive noise to the total noise of device is rather complex and challenging.

In this work, a magnetostrictive deformation calculation technique is proposed which is based on a Finite Element (FE) approach. A two-dimensional deformation of the core is then calculated by using the magnetostriction measurement results of the core material. The measurements have been performed by using a dual heterodyne laser setup which has been developed in the Electrical Energy Laboratoty (EELAB) of Ghent University. More about the setup and the measurement results can be found elsewhere [7], [8]. The method is then applied to calculate the deformation of a test setup which is a single-phase transformer. To validate the FE calculation results, vibration measurements have been carried out for the same test transformer setup. The vibration measurements were performed using a laser scanning vibrometer. The comparison of the results of the two will be presented in this work.

The manuscript is organised as follows. In the next section the common transformer assembly and the test transformer core design will presented. After that, the FE computation technique and the vibration measurement will be presented. The calculated and the measured deformation results of the single-phase test transformer core will be presented and discussed at the end.

\section{Test transformer assembly}

Grain-oriented (GO) electrical steel is often used in the cores of transformers. Compared with nonoriented (NO) electrical steel type, the GO materials can normally obtain a higher induced magnetic induction $B[\mathrm{~T}]$ for the same applied magnetic field $H[\mathrm{~A} / \mathrm{m}]$. However, this may only be valid for the rolling direction of the material, where the grains are improved. Therefore, for the assemblies of transformers a lap-joint design, overlapping of the laminations in the corners of the core, is preferred. In this way, the flux passes almost always along the rolling direction of the material. It is commonly known that in the joint corners the flux travels from one lamination to another through the small air gap between the two in the out-of-plane direction. This in turn creates an attractive interlaminar force in the corners which results in a clamping noise [2], [3], [9].

Considering the extra noise of the lap-joint corners, the test transformer studied in this work has laminations cut in one piece without any joint corners. Such design is selected to resemble only the magnetostriction noise and avoid any other noise sources. For the selection of the core material, a GO material was not beneficial since such material is strongly isotropic. To this end, a NO material type M350-50A was applied. This material has a thickness of $0.5 \mathrm{~mm}$ and $3.50 \mathrm{~W} / \mathrm{kg}$ losses at $1.5 \mathrm{~T}$ and $50 \mathrm{~Hz}$ magnetisation. The test transformer laminations are cut by using spark erosion technique to minimize any damages to the magnetic properties of the material. The core is $30 \mathrm{~cm} \times 30 \mathrm{~cm}$ and has a thickness of $2.5 \mathrm{~cm}$ made out of a stack of 50 laminations. Fig. 1 shows the test transformer setup which is wounded with the magnetisation winding over three legs (L2, L3 and L4) and the last leg (L1) is left free for the vibration measurements. 


\section{The magnetostrictive deformation: calculation ver- sus measurement}

\subsection{The computation technique}

The proposed technique makes use of program in-house developed in EELAB. It is based on the FE approach with a magnetostrictive model of the material. This model is devised using an artificial neural network of the magnetostriction behaviour of the material. These networks are trained based on the magnetostriction strain measurement results of the core material as a function of magnetic induction $B[\mathrm{~T}]$. The strain measurements have been performed by using a dual heterodyne laser setup. The two-dimensional behaviour of the material in parallel and perpendicular direction to the magnetic flux is measured, $\lambda_{\|}$ and $\lambda_{\perp}$ respectively. The samples are magnetised under a sinusoidal waveform with $50 \mathrm{~Hz}$ frequency. Looking at the frequency response of the data, the harmonics higher than four times the base frequency are small and can be neglected. Since only the even harmonics are relevant to the magnetostrictive noise, a database of the $100 \mathrm{~Hz}$ and $200 \mathrm{~Hz}$ harmonics is made [10]. The artificial neural network models are made for the $\lambda_{\|}$and $\lambda_{\perp}$. Although the core material is a NO electrical steel still some degree of anisotropy exists. Thus, looking at the test transformer, the flux in two legs is along the rolling direction of the material and in the other two legs along the perpendicular direction. As a result, two sets of samples, cut along and perpendicular to the rolling direction, are measured and modelled. In the FE calculations the small anisotropy is taken into account then.

The technique calculates the magnetic induction $B[\mathrm{~T}]$ value for every mesh triangle of the core in the time domain. After that the program calculates the magnetostrictive strains $\lambda_{\|}$and $\lambda_{\perp}$ for every magnetic induction $B[\mathrm{~T}]$ value by using the models. In the final step the vibrations and deformation of the core are calculated based on the mechanical properties of the core. The calculations are performed for the nodes shown in Fig. 1 under a magnetisation of $1.2 \mathrm{~T}$.

\subsection{The vibration measurements}

As a validation, the vibrations and deformations of the same nodes are measured by using a PSV-400 laser scanning vibrometer. The vibration measurements have been performed at the Acoustics and Vibration Laboratory at the Free University of Brussels. The scanner has multiple resolution ranges and it has been adjusted for $0.2 \mathrm{~mm} / \mathrm{s} / \mathrm{V}$.

A magnetisation signal is sent from a PC based program to a PAS1000 amplifier and then sent to the magnetisation winding around the core. Vibration of the core was then measured by the scanning vibrometer and post-processed to calculate the displacements. The magnetising voltage is adjusted to create a sinusoidal magnetic induction $B$ of $1.2 \mathrm{~T}$ in the core. To avoid any frictions of the transformer core with the underlying surface, the core was hung. To this end, the core was in a horizontal plane parallel to the ground which makes the core weight to be evenly distributed. The laser head was pointed at the free leg of the transformer, as shown in Fig. 2. As a comparison with calculated results, single point vibration measurements have been performed for the same nodes. 


\section{Results and discussion}

The magnitude and phase of the $100 \mathrm{~Hz}$ and $200 \mathrm{~Hz}$ harmonics of the displacement data of the three selected nodes obtained by the calculation and the measurement techniques are shown in Table 1. Both the vibration measurement results and the FE results show that the $100 \mathrm{~Hz}$ harmonic component magnitude is relatively larger than that of the $200 \mathrm{~Hz}$ and thus the prime defines the deformation of the nodes.

Looking at the $100 \mathrm{~Hz}$ data, for node 1 there is a good agreement between the FE calculations and the vibration measurements. The FE calculation results show the same deformations along the free leg. However, the measurement results show that the core deforms less in the middle point, node 3 , while it deforms more in the corners. To visualize the decrease of the deformation toward the mid leg, a three-dimensional animation of the surface deformation of the core free leg has been visualized. Fig. 3 shows two pictures of the animation of the $100 \mathrm{~Hz}$ component, when the free leg shows the maximum and the minimum magnetostrictive deformations from top to the bottom, respectively. The deformation of the free leg is clearly shown in these pictures.

In addition, a two-dimensional FE modal analysis for determining the eigen modes and natural frequencies has been performed. The first nine vibration eigen modes (EM1 to EM9) of the core (i.e. with the lowest natual frequencies) are shown in Fig. 4. Looking at these modes, one could conclude that the fourth and the fifth eigen modes create the aforementioned effect seen in the vibration measurements of the free leg. The fact that this effect is not seen in the calculated vibrations may be due to the deviation of the higher order of the eigen modes. These modes are more dominant in the measurements which can be due to the friction between the different laminations of the core.

\section{Conclusion}

Determining the magnetostrictive deformation of transformer cores is rather challenging. In this work a calculation technique is proposed which is based on the finite element technique with an artificial neural network of the core material. The technique is applied to calculate the deformation of a single-phase test transformer for three nodes. As a validation, the vibration of the same core is measured at the same nodes by using a laser scanning vibrometer. The results show good agreement for one node and less in the other two nodes, for which some possible reasons are discussed. However, the technique can still be improved by going from a two-dimensional approach to a three-dimensional approach.

\section{References}

[1] L. Vandevelde, J. Melkebeek, Magnetic forces and magnetostriction in electrical machines and transformer cores, IEEE Trans. Magn., 2003, vol. 39, no. 3, pp. 1618-1621

[2] B. Weiser, A. Hasenzagl, T. Booth and H. Pfützner, Mechanisms of noise generation of model transformer cores, Journal of Magnnetism and Magnetic Material, vol. 160, 1996, pp. 207-209

[3] B. Weiser, H. Pfützner and J. Anger, Relevance of Magnetostriction and Forces for the Generation of Audible Noise of Transformer Cores, IEEE Trans. Magn., 2000, vol. 36, no. 5, pp. 3759-3777 
[4] T. Nakata, N. Takahashi, M. Nakano, K. Muramatsu and M. Miyake, Magnetostriction Measurements with a Laser Doppler Velocimeter, IEEE Trans. Magn., 1994, vol. 30 , no. 6 , pp. $4563-4565$

[5] P.I. Anderson, A.J. Moses and H.J. Stanbury, An automated system for the measurement of magnetostriction in electrical steel sheet under applied stress, Journal of Magnnetism and Magnetic Material, 2000, vol. 21, pp. 714716

[6] Y. Kai, Y. Tsuchida, T. Todoka and M. Enokizono, Measurement of two-dimensional magnetostriction and the vector magnetic property for a non-oriented electrical steel sheet under stress, Journal of Applied Physics, 2012, vol. 111, no. 7, 07E320

[7] S. Gorji Ghalamestani, T. Hilgert, L. Vandevelde, J. Dirckx and J. Melkebeek, Magnetostriction Measurement by Using Dual Heterodyne Laser Interferometers, 2010, IEEE Trans. Magn., vol. 46, pp. 505-508

[8] S. Gorji Ghalamestani, L. Vandevelde, J. Dirckx and J. Melkebeek, Magnetostriction and the Influence of Higher Harmonics in the Magnetic Field, IEEE Trans. Magn., 2012, vol. 48, no. 11, pp. 3981-3984

[9] H. Pfützner, E. Mulasalihovic, H. Yamaguchi, D. Sabic, G. Shilyashki and F. Hofbauer, Rotational Magnetization in Transformer Cores-A Review, IEEE Trans. Magn., 2011, vol. 47, no. 11, pp. 4523-4533

[10] T. Hilgert, L. Vandevelde and Jan. Melkebeek, Comparison of magnetostriction models for use in calculations of vibrations in magnetic cores, IEEE Trans. Magn., 2008, vol. 44, no. 6, pp. 874-877 


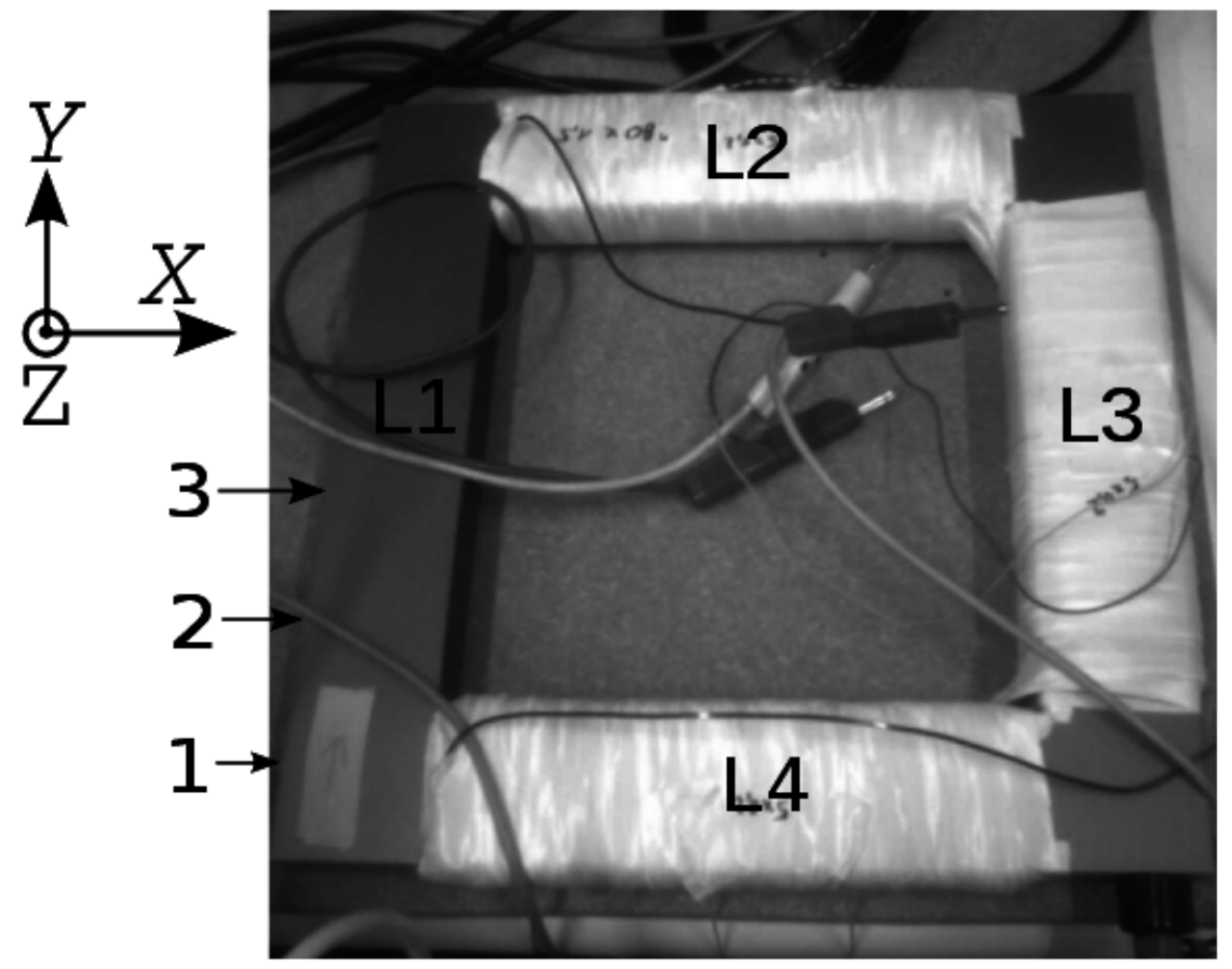

Figure 1: 


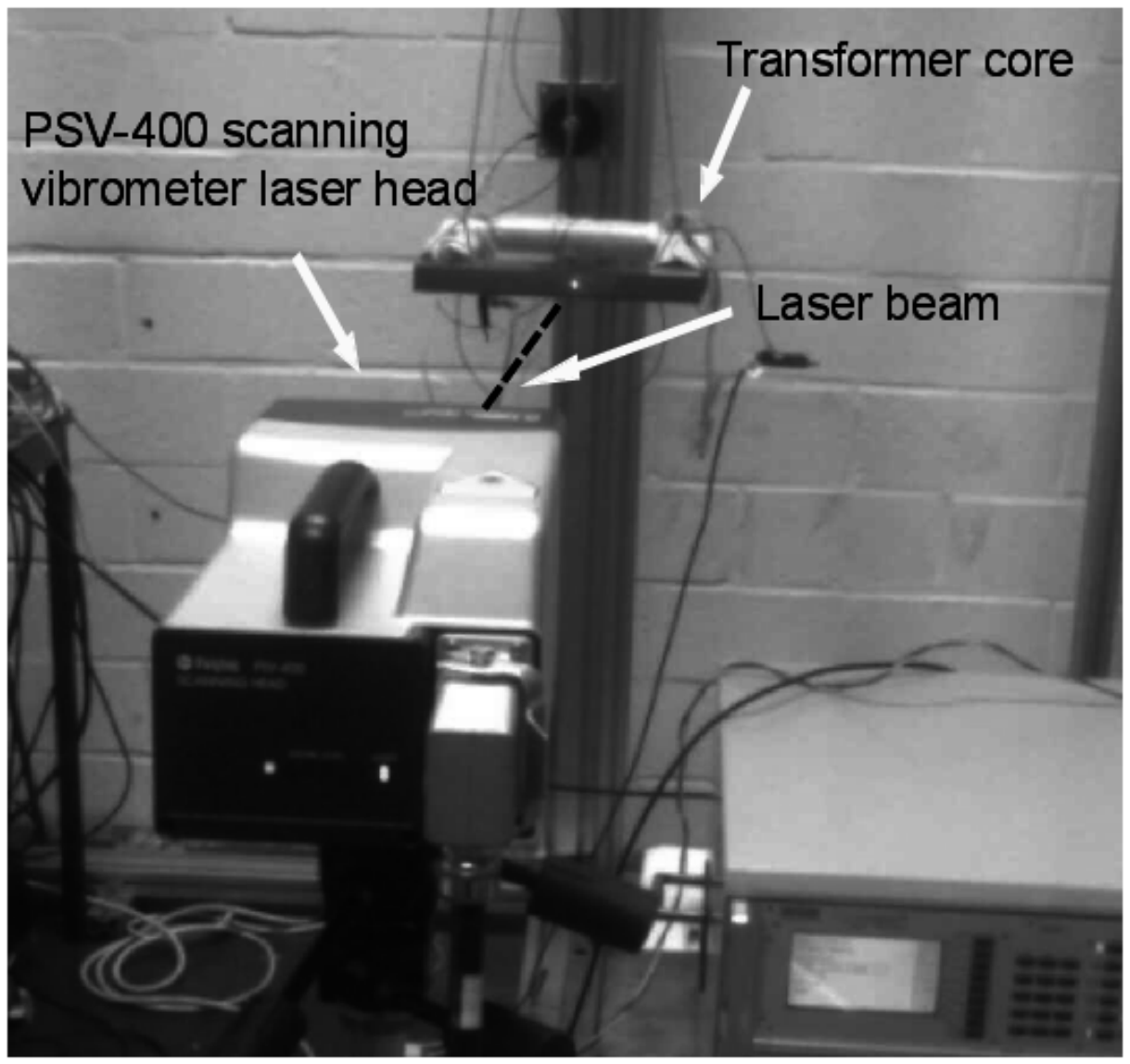

Figure 2: 

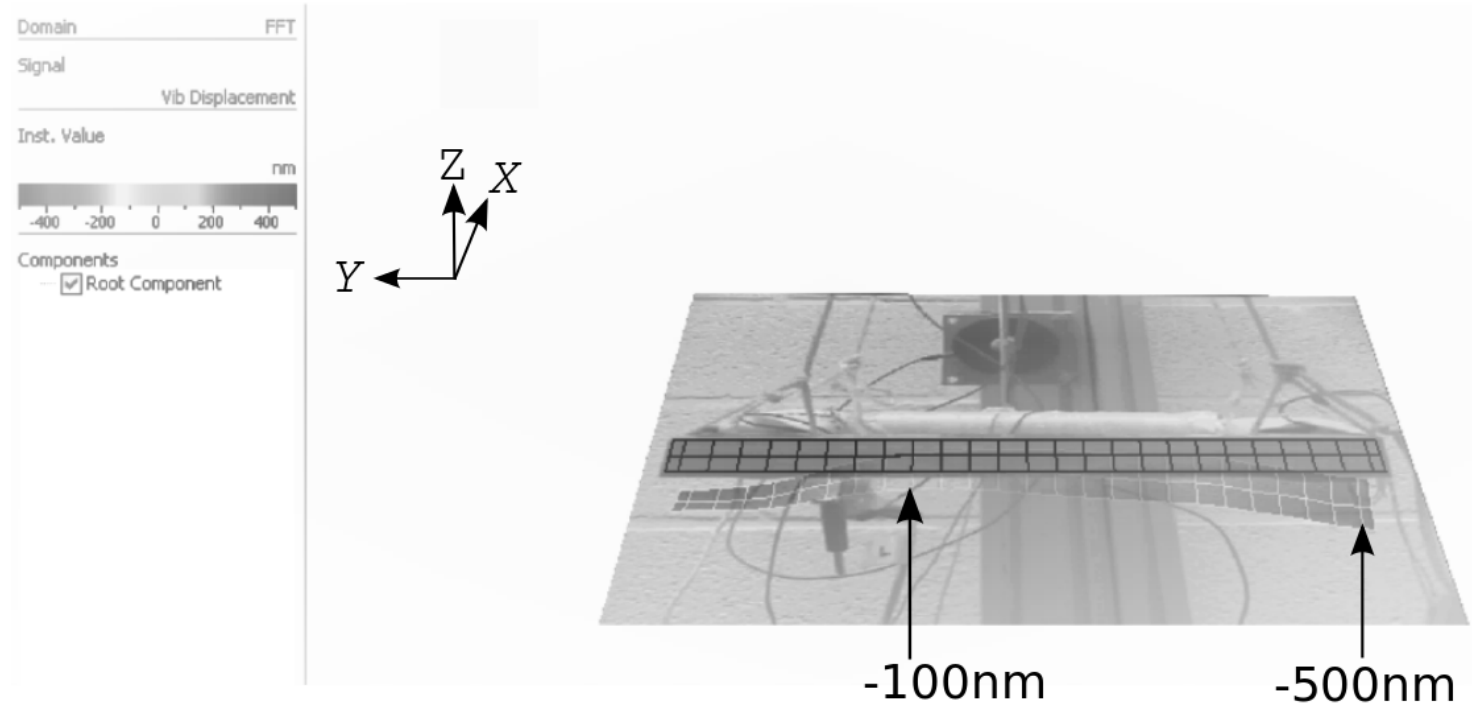

(a)

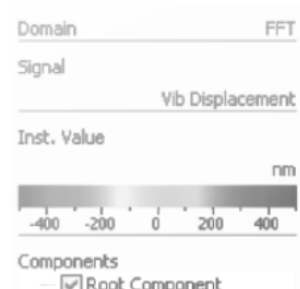

$Y \overbrace{}^{Z}$

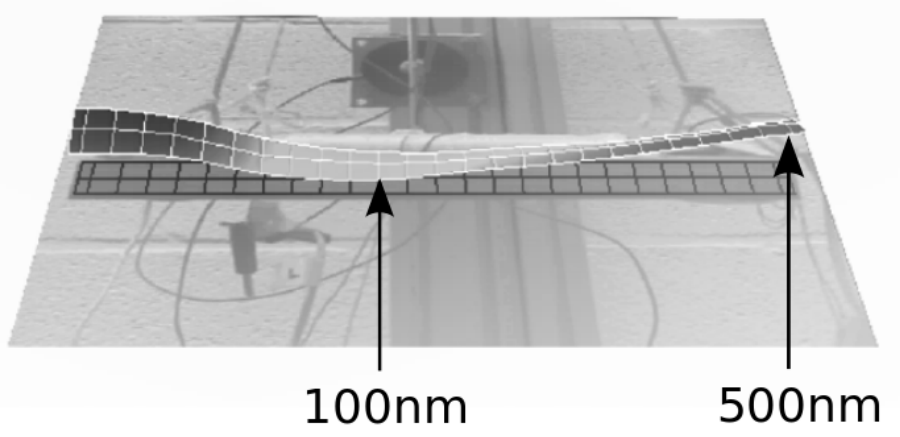

(b)

Figure 3: 


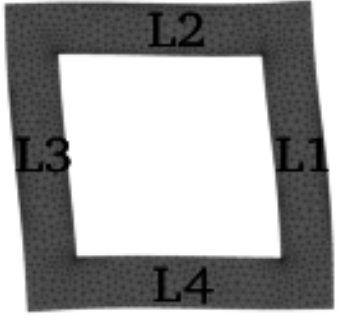

EM1

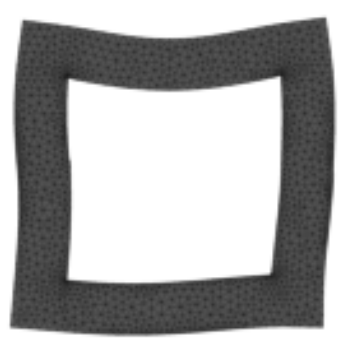

EM4

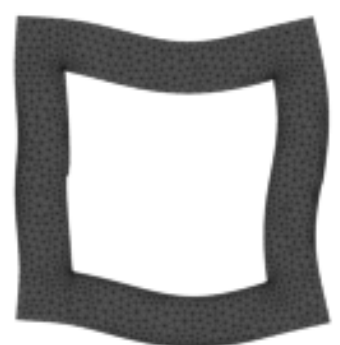

EM7

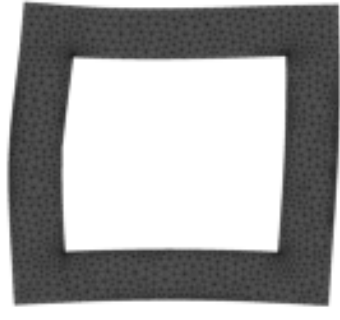

EM2

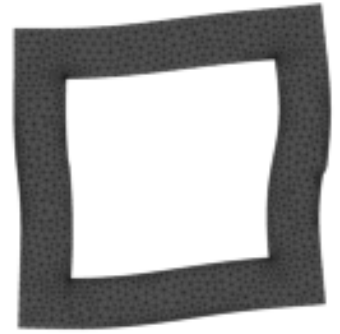

EM5

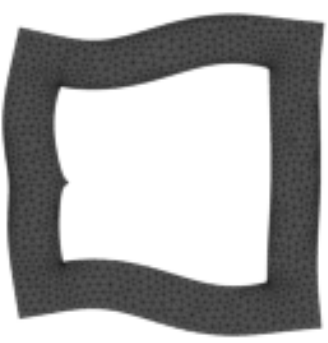

EM8

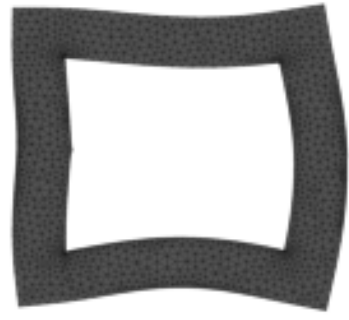

EM3

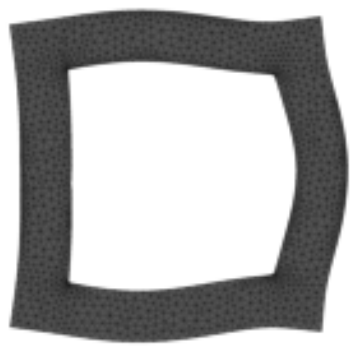

EM6

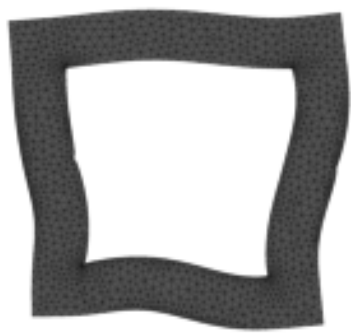

EM9

Figure 4: 
Table 1: The $100 \mathrm{~Hz}$ and $200 \mathrm{~Hz}$ magnitude [m] and phase spectrum [rad] displacement data of the selected nodes calculated by the FE technique and measured by the scanning laser vibrometer

\begin{tabular}{|c|c|c|c|c|}
\hline & \multicolumn{2}{|c|}{ FE calculation results } & \multicolumn{2}{c|}{ vibration measurement results } \\
\hline node & $100 \mathrm{~Hz}$ & $200 \mathrm{~Hz}$ & $100 \mathrm{~Hz}$ & $200 \mathrm{~Hz}$ \\
\hline 1 & $3.67 \mathrm{E}-7 \angle 0.06$ & $1.88 \mathrm{E}-8 \angle-2.18$ & $3.81 \mathrm{E}-7 \angle-0.23$ & $4.59 \mathrm{E}-8 \angle-2.61$ \\
\hline 2 & $3.59 \mathrm{E}-7 \angle 0.05$ & $1.74 \mathrm{E}-8 \angle-2.10$ & $2.07 \mathrm{E}-7 \angle-0.08$ & $5.33 \mathrm{E}-8 \angle 1.97$ \\
\hline 3 & $3.59 \mathrm{E}-7 \angle 0.05$ & $1.76 \mathrm{E}-8 \angle-2.11$ & $1.30 \mathrm{E}-7 \angle-0.32$ & $6.30 \mathrm{E}-8 \angle 1.58$ \\
\hline
\end{tabular}




\section{List of Figures}

1 The test transformer core showing the nodes where the FE calculations $\begin{array}{ll}\text { and the vibrations measurements are performed and the coordinate system } & 6\end{array}$

2 Vibration measurement of the test transformer core by using a PSV-400 vibrometer Polytec laser . . . . . . . . . . . . . . . . 7

3 The instance when the core free leg shows the the maximum (outward) deformation (top) and minimum (inward) deformation (bottom) . . . . . 8

4 The first nine vibration eigen modes of the transformer core . . . . . . . 9 


\section{List of Tables}

1 The $100 \mathrm{~Hz}$ and $200 \mathrm{~Hz}$ magnitude [m] and phase spectrum [rad] displacement data of the selected nodes calculated by the FE technique and measured by the scanning laser vibrometer . . . . . . . . . . . . . . . . . 10 